Article

\title{
Design and Implementation of a Multi-Band Active Radar Calibrator for SAR
}

\author{
Liang Li 1,2,3,*, Gukun Liu 1,2,3, Jun Hong ${ }^{1,2,3}$, Feng Ming 1,2 and Yu Wang 1,2 (D) \\ 1 Institute of Electronics, Chinese Academy of Sciences, Beijing 100190, China; \\ liuguikun@std.uestc.edu.cn (G.L.); jhong@mail.ie.ac.cn (J.H.); fming@mail.ie.ac.cn (F.M.); \\ wangyu@mail.ie.ac.cn (Y.W.) \\ 2 National Key Laboratory of Sciences and Technology on Microwave Imaging, Beijing 100190, China \\ 3 University of Chinese Academy of Sciences, Beijing 100049, China \\ * Correspondence: liliang@mail.ie.ac.cn; Tel.: +86-10-58887665
}

Received: 29 April 2019; Accepted: 23 May 2019; Published: 1 June 2019

\begin{abstract}
Over the past decade, IECAS (Institute of Electronics, Chinese Academy of Sciences) has developed a set of L-, S-, C-, and X-band active radar calibrators that are deployed during the calibration campaigns for HJ1C synthetic aperture radar (SAR), Gaofen-3 SAR, and so on. In the near future, P-band and Ka-band spaceborne SARs will be launched. We found that it is not convenient to develop special active radar calibrators (ARCs) for a specific SAR or a specific frequency band SAR, and the acquired experience could help in the design and development of a multi-band ARC. This paper describes the design and implementation of a multi-band active radar calibrator which can operate in the L-, C-, X-, and Ka-bands. Moreover, laboratory measurements are performed to characterize the performance of the multi-band ARC, paying particular attention to the gain stability, the system transfer function, the gain flatness, and the linearity of the ARC receiver. Three such ARCs are developed, and to our knowledge, the multi-band ARC is the first of its kind in China or even in the world, and it can be used to implement the calibration campaigns of the Chinese Gaofen-3 SAR, Shenzhen-1 SAR, Luojia-2 SAR, and so on.
\end{abstract}

Keywords: multi-band; transponder; active radar calibrator; calibration; SAR

\section{Introduction}

Synthetic aperture radars (SARs) are an important remote sensing tool for the acquisition of quantitative information about the Earth's environment. Nowadays, there are L-, S-, C-, and X-band spaceborne SARs in orbit. Additionally, lower P-band and higher Ka-band spaceborne SARs will be launched in the near future. For quantitative application, external calibration should be executed, and the active radar calibrator (ARC) plays an important role in the external calibration of SAR instruments due to its adjustable radar cross section (RCS), capability of changing its internal delay, high RCS, and small size. So, ARCs have been developed for many famous spaceborne SARs such as Radarsat-1/2, TerraSAR, Sentinel, and Gaofen-3 [1-7].

Most of the existing ARCs operate in a single band. For example, the ARCs developed for Radarsat-1/2, Sentinel, ERS-1, and Gaofen-3 operate in the C-band [1,3,4,6,8], the ARCs developed for TerraSAR operate in the X-band [2], the ARCs developed for ALOS/PALSAR operate in the L-band [9], and the ARC developed for Chinese HJ1C operates in the S-band [10]. Even for multi-band SAR systems, the ARCs are also developed as single-band instruments. For instance, the NASA/JPL DC-8 aircraft SAR operated at three frequencies (L-, C-, and P-bands), but the ARCs used for calibration operated at a single frequency (three L-band ARCs and three C-band ARCs were used) [11]. Another 
SIR-C/X-SAR operated in the L-, C- and X-bands; however, two L-band ARCs, two X-band ARCs, and three C-band ARCs were used for the calibration of the SIR-C/X-SAR [12].

Single-band ARCs have been developed for SAR systems, even for multi-band SAR instruments. However, with the increase of spaceborne SARs, it is not convenient to develop special ARCs for a specific SAR or a specific frequency band SAR. Therefore, the concept of a new multi-band ARC for spaceborne radar calibration is proposed in this study. Of course, it is very difficult to develop such an ARC, especially when it has many operational frequency bands. Many key technologies such as multi-band antenna, multi-band gain stability, and a large receiver dynamic range must be implemented for the development of the multi-band ARC. We took many measures and overcame these key technologies to design and develop three multi-band ARCs, which work in the L-, C-, X- and Ka-, operational bands. The specifications such as RCS stability, RCS ripper, linearity, and dynamic range were confirmed by the measurements. The three multi-band ARCs will be used to implement the calibration campaigns of Chinese Gaofen-3 SAR, Shenzhen-1 SAR, Luojia-2 SAR, and so on.

\section{Materials and Methods}

\subsection{Principles of $A R C$}

The RCS of an ARC is given by Equation (1) [11]:

$$
\sigma=\frac{\lambda^{2}}{4 \pi} G_{a} G_{T} G_{R}
$$

where $G_{T}$ and $G_{R}$ are the gain of the transmitting and receiving antennas of ARC, respectively; $G_{a}$ is the gain of the radio frequency $(\mathrm{RF})$ channels inserted between the receiving and transmitting antennas to increase the RCS; and $\lambda$ is the radar wavelength.

It can be seen from Equation (1) that the RCS of ARC depends on the gain of the RF channel and the TX and RX antenna gain. Actually, the gains of the receiving antenna and the transmitting antenna are the same. The RCS can be high enough in theory if either the RF channel gain or the antenna gain, or both, is large enough. However, they are restricted by each other. The higher the antenna gain is, the bigger its size is. It is very hard to keep the ARC at a reasonable size, and it is very heavy due to the bigger structure of the antennas, which then have pointing uncertainty and deployment issues. If the $\mathrm{RF}$ channel gain is designed to be very high, on the one hand, the gain stability requirements cannot be satisfied, and on the other hand, the isolation between the receiving and transmitting antennas is difficult to satisfy $[13,14]$. Thus, many factors should be considered such as the value of RCS, the size and weight of the ARC, the stability of RCS, and so on.

The level of the receiving signal is also a precondition for the design of an ARC. For a certain RCS, the higher the signal level reaching the ARC is, the higher the output level of the ARC is. Therefore, the demands such as saturated output power, a $1 \mathrm{~dB}$ compression point, and linearity for the final amplifier are very high. The received signal level of the ARC can be given according to the radar equation $[15,16]$ :

$$
P_{r}=\frac{\lambda^{2}}{(4 \pi R)^{2}} \cdot P_{E I R P} \cdot G_{R}
$$

where $P_{E I R P}$ is effective the isotropic radiation power (EIRP) of SAR, and $R$ is the distance between SAR and ARC. The distance is decided by the orbit and the incident angle of SAR. The multi-band ARC was designed for several SARs working in the L-, $C_{-}, X_{-}$, and Ka-bands. The received signal level will be much different even for SARs in the same band, because the EIRP, orbit, and incident angle will be different. Therefore, the ARC is required to have a large dynamic range.

So, we should calculate the received signal level first and then deduce the output power of ARC according to the RCS. The final amplifier of ARC can be decided based on the output power, and the ARC can be designed, including the antenna size, the gain of RF channels, and so on. 


\subsection{The multi-band ARC}

In the framework of future SAR ground calibration campaigns, three equal ARCs will be deployed, covering the four operational bands ( $\backslash \mathrm{C} \backslash X \backslash \mathrm{Ka}$ ) of the SAR sensors. Figure 1 shows the block diagram of the ARC. It consists of an antenna and servo subsystem, a radio frequency (RF) subsystem, a control and data collection subsystem, and a remote-controlled subsystem.

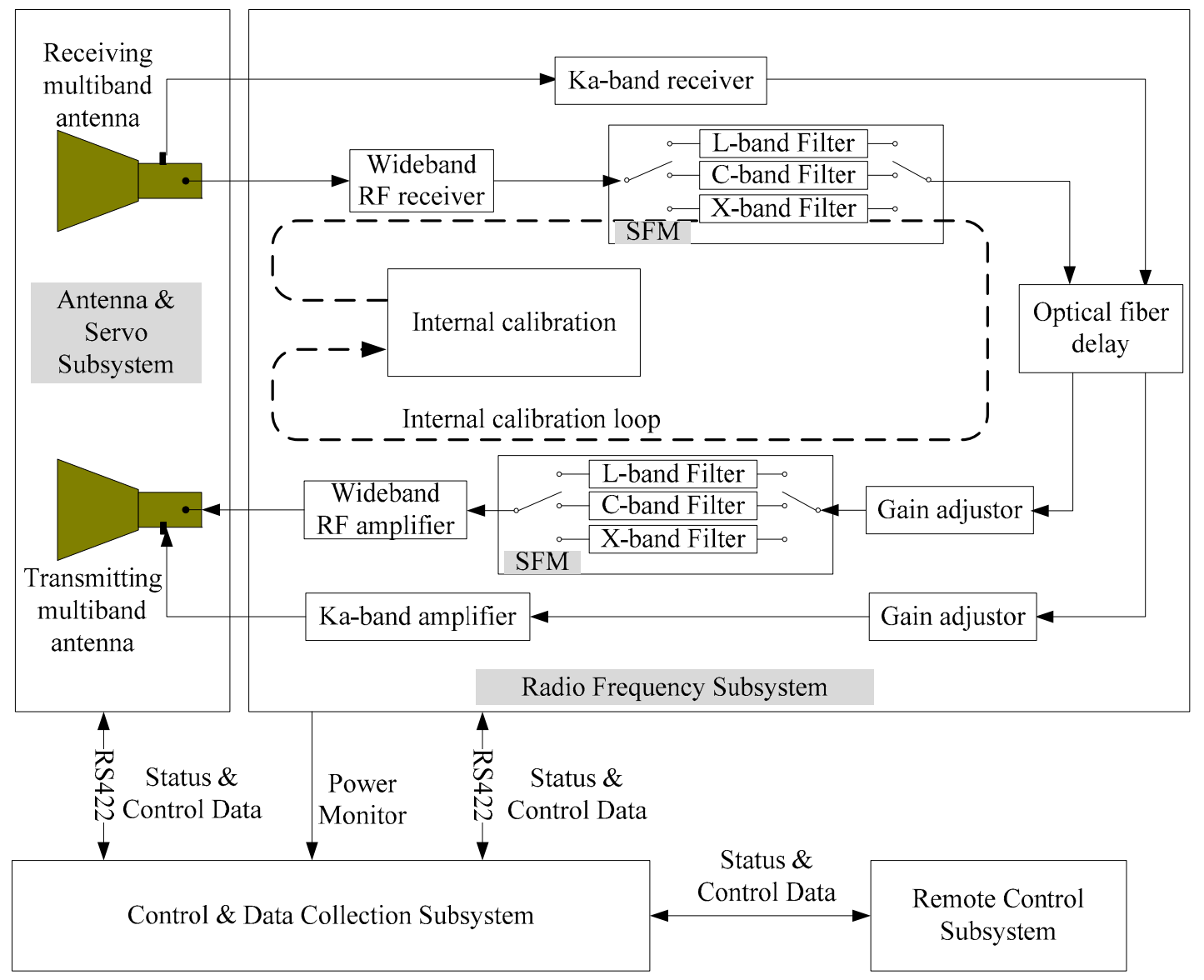

Figure 1. The block diagram of the active radar calibrator (ARC).

The antenna and servo subsystem comprises two similar multi-band antennas used for reception and transmission, and the servo allows the ARC to move mechanically in the elevation and azimuth directions. The RF subsystem includes a wideband RF receiver, a Ka-band receiver, an optical fiber delayer, a gain adjustor, a wideband RF amplifier, a Ka-band amplifier, an internal calibration module, and a switched filter module (SFM). It amplifies the signal level that complies with the required RCS value and selects the work frequency, delay, and so on. The control and data collection subsystem is used to control the whole ARC and collect the SAR signal from the radio frequency subsystem. The remote-controlled subsystem is in charge of monitoring, controlling, and scheduling the ARC. This subsystem is located away from the ARC, and its communication method is the $4 \mathrm{G}$ communication network. In the following text, we emphasize the design of the antenna and radio frequency system. 


\section{Results}

\subsection{Design of the ARC}

\subsubsection{Antenna Design}

The form and performance of the antenna determine the whole structure and performance of the ARC to some extent. Considering the operation at a multi-band frequency of one ARC, the antennas must be able to work at a frequency range from the L-band to Ka-band. It is almost impossible to design an ultra-wideband antenna that works from the L-band to the Ka-band with the specifications of gain, a $3 \mathrm{~dB}$ beam width, an antenna pattern, and isolation between receiving and transmitting antenna to satisfy the requirements for the ARC at all four bands. So, a multi-band antenna needed to be designed for the ARC. We designed a combined antenna consisting of an L/C-band common aperture horn antenna $[17,18]$, an X-band dual-polarimetric corrugated horn antenna, and a Ka-band corrugated horn antenna for the multi-band ARC. A schematic illustration of the combined antenna is shown in Figure 2. The types of polarization used are H-pol for the L-band, H- and V-pol for the Cand X-bands, and V-pol for the Ka-band.

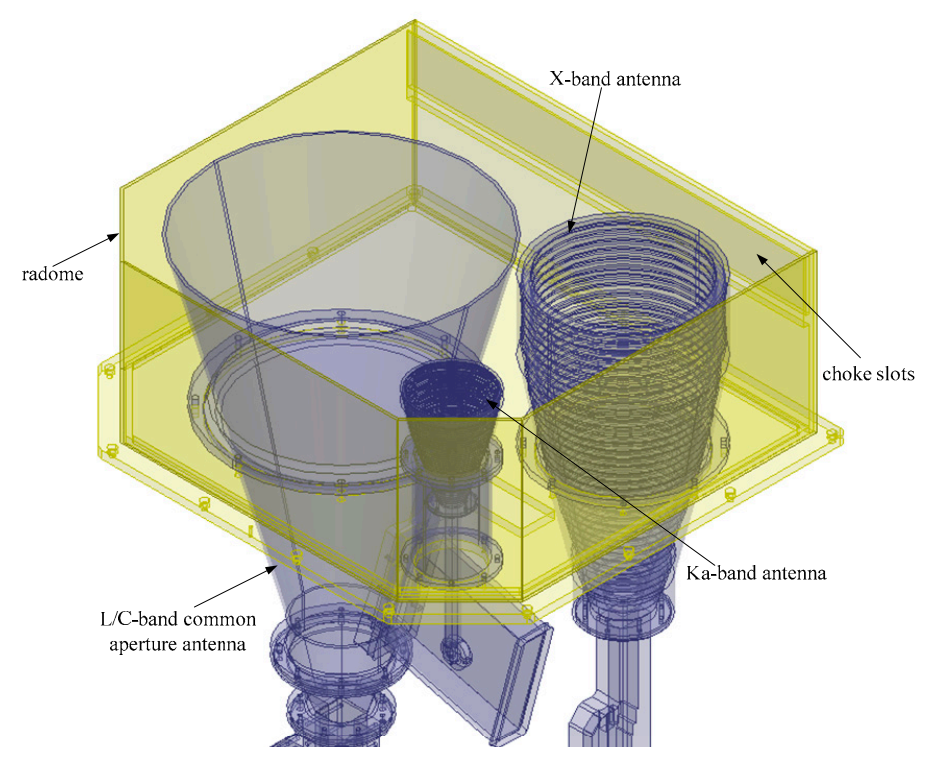

Figure 2. Schematic illustration of the combined antenna.

There are many key technologies and difficulties in the design of the multi-band antenna such as L/C-band common aperture design, C/X-band dual-polarimetric and high polarization isolation design, L-band miniaturization design, and so on. The dual-polarimetric and high polarization isolation design technology has been introduced in existing literature [6,19]. We emphasize the key technology of L/C-band common aperture design. A schematic illustration of the L/C-band common aperture antenna is shown in Figure 3. 


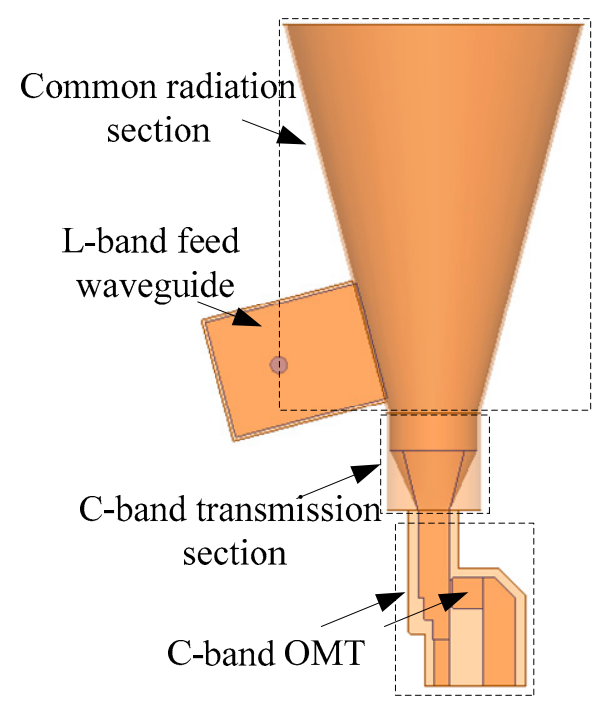

Figure 3. Schematic illustration of the L/C-band common aperture antenna. OMT: ortho-mode transducer.

The L/C-band common aperture antenna consists of a common radiation section, an L-band feed waveguide, a C-band transmission section, and an ortho-mode transducer (OMT). The amplitude and phase distribution of the aperture field will affect the antenna's radiation performance, so the structure design of the common radiation section is very important. Generally speaking, the feed port of an antenna is located at the transmission section. However, it can be seen from Figure 3 that the L-band feed waveguide is placed at the common radiation section, which reduces the length of the antenna significantly.

In addition, the demand for isolation between the receiving antenna and transmitting antenna is very high in order to ensure precision in the RCS of the ARC. In general, the isolation should be at least $25 \mathrm{~dB}$ better than the gain of the radio frequency circuit [14]. In order to satisfy the demand, on the one hand, the level at 90 degree from the boresight should be designed to be as low as possible, and on the other hand, choke slots were placed on the sidewall of the radome. Based on the measures above, the isolation reaches $79 \mathrm{~dB}$ for the L-band, $80 \mathrm{~dB}$ for the C-band, $82 \mathrm{~dB}$ for the $\mathrm{X}$-band, and $84 \mathrm{~dB}$ for the Ka-band, based on measurement in an open space. The isolation is very high and it is hard to measure. First we chose an open space and the Tx and Rx antennas pointed up to the sky in the measurement, and then the IF band of vector network analyzer was set to $100 \mathrm{~Hz}$.

The gains designed on respective center frequencies are $12 \mathrm{~dB}$ for the L-band, $22 \mathrm{~dB}$ for the C-band, $23 \mathrm{~dB}$ for the $\mathrm{X}$-band and $27 \mathrm{~dB}$ for the Ka-band, with a typical sidelobe level of $-17 \mathrm{~dB}$ for the four bands, which minimizes multipath effects and background noise.

\subsubsection{RF Subsystem Design}

The RF subsystem is very important, and many functions and performance aspects of ARC are realized based on the RF subsystem. The scheme of the RF subsystem is depicted in Figure 1. It consists of a receiver, optical fiber delay, a gain adjustor, an amplifier, an internal calibration module, an SFM, and so on.

Based on the demands for the RCS of the ARC, the gains are about $54 \mathrm{~dB}$ for the L-band, $47 \mathrm{~dB}$ for the $\mathrm{C}$-band, $50 \mathrm{~dB}$ for the $\mathrm{X}$-band, and $59 \mathrm{~dB}$ for the Ka-band at maximum, and this can be reduced by $15 \mathrm{~dB}$ in $5 \mathrm{~dB}$ steps. The bandwidth is $200 \mathrm{MHz}$ for the L-band, $240 \mathrm{MHz}$ for the C-band, $1200 \mathrm{MHz}$ for the X-band and $1200 \mathrm{MHz}$ for the Ka-band.

The multi-band ARC demands that the RF subsystem should be able to work from the L-band to the Ka-band. Furthermore, specifications such as gain stabilization and gain flatness are very high.

- RF channel 
Based on the value of RCS and the gain of the antenna, it is can be deduced from Equation (1) that the gain of the RF channel should be about $54 \mathrm{~dB}$ for the L-band, $47 \mathrm{~dB}$ for the C-band, $50 \mathrm{~dB}$ for the $\mathrm{X}$-band, and $59 \mathrm{~dB}$ for the Ka-band at maximum. As is commonly known, many attenuating devices such as attenuators used for impedance matching, filters used to filter the useless signals and so on are situated in the RF channel, so the total gain of amplifiers will be far higher than the gain of the RF channel. Questions of stabilization and gain flatness will arise subsequently.

Technology using a multistage and adjustable equalization filter was utilized to ensure the gain flatness. The cavity filter shown in Figure 4 with some tuning rods was used somewhere in the RF channel. The tuning rods were tuned until the gain flatness satisfied the requirement, and then they were fixed.

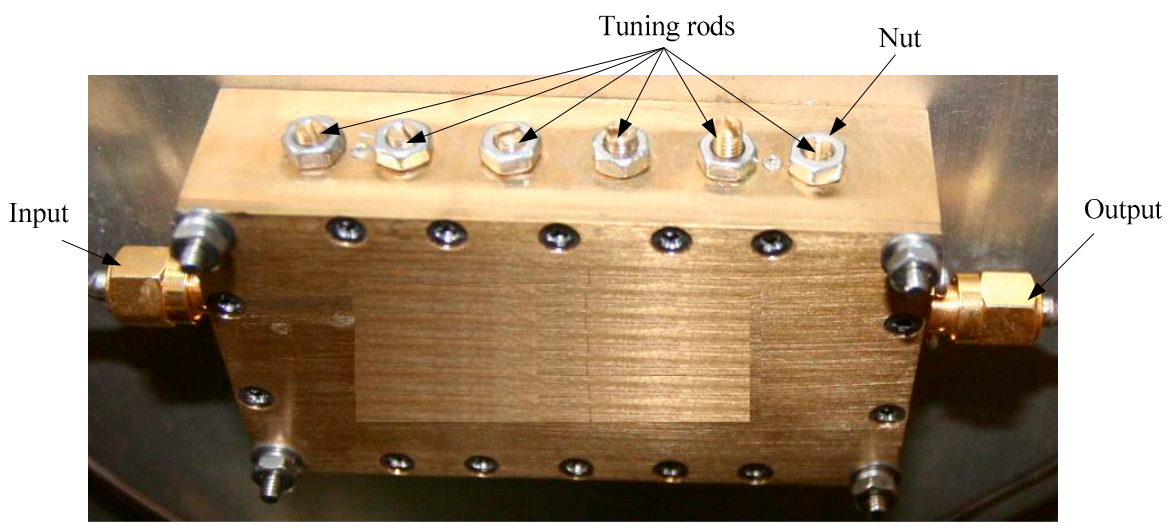

Figure 4. Example of the cavity filter with some tuning rods.

Moreover, two SFMs were used to attenuate out-of-band spurious signals. For example, if the ARC was used to calibrate the SAR operating at C-band, the SFMs would be switched to the C-band filter. This can attenuate out-of-band spurious signals and reduce the thermal noise, and then the signal-to-noise ratio can be improved.

- Fiber optic delay module

There many methods that can be used to achieve tunable delay, such as optical fiber delay, bulk acoustic wave (BAW), surface acoustic wave (SAW) delay, digital delay, and so on. An optical fiber delay unit was chosen because of its advantages of no frequency translation being required, low loss, and a wide bandwidth. However, the optical fiber is temperature-sensitive, and its consequent amplitude instability should be paid sufficient attention. A temperature control measure was taken in the optical fiber delay unit, and a special design for the structure of the optical fiber was determined.

The fiber optic delay module can move the point target response in the final image to a low-backscattering background. For example, when the ARC is placed near water surfaces, through fiber optic delay, the echo return can be delayed as if the ARC was located on the water where the background noise is low. This greatly reduces the demands for the calibration site. Furthermore, fiber optic delay can mean that the point target response in the final image is not at the location of the ARC, which reduces the re-radiation effects of the structure itself; therefore, a higher signal-to-clutter ratio and better calibration accuracy can be achieved [20]. Furthermore, distortion due to antenna leakage is non-coherent after fiber optic delay and can be suppressed with range compression [21]. Three optical fibers with the length of 0.5 us, 0.5 us and 1 us were used between laser and detector, and the delay was tuned through the optical switches.

- Gain calibration circuit

The gain stability is achieved by the gain calibration circuit, as depicted in Figure 5. A series of calibration pulses are fed to the circuit near its input through CP1. These go around the main RF circuit, with a 1 or $2 \mu$ s delay, and they are then detected at detector $C$. The original pulse without 
the delay is also detected at the same detector in the gain calibration module. The amplitudes of the delayed and direct pulses are compared, and any error nulled using the voltage control attenuator in the main RF circuit. The RF unit gain is therefore stabilized. Gain calibration module, the couplers CP1 and CP2 are housed in a weatherproof enclosure, which is maintained at $55 \pm 2{ }^{\circ} \mathrm{C}$. Thus, the gain stability is independent of the surrounding temperature. In this way, the gain of the ARC is tied to the gain of the calibration module, which only includes highly-stable passive components and is housed in a constant-temperature box. So, the gain stability can be rather good.

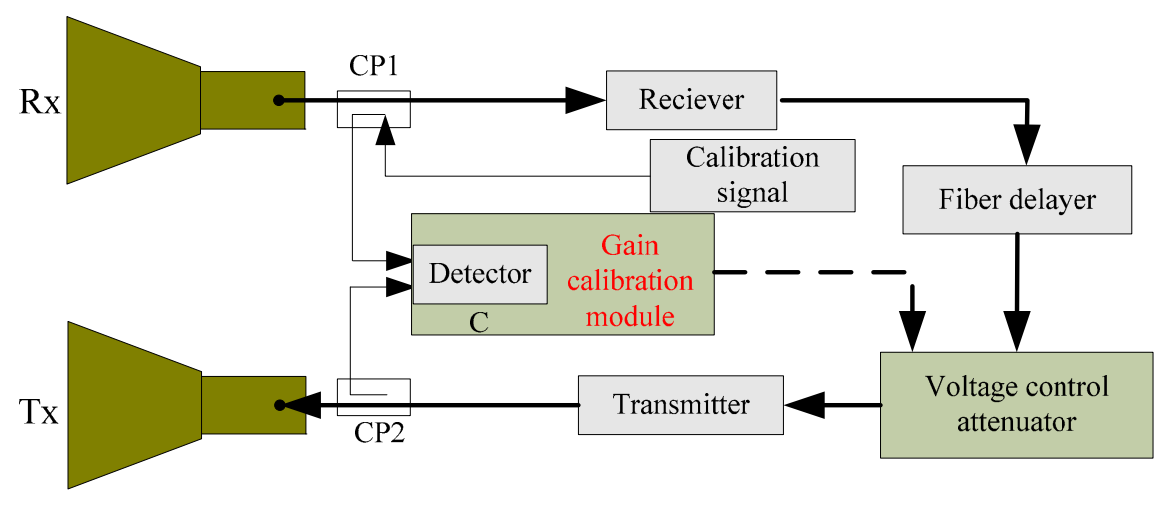

Figure 5. Gain calibration circuit scheme.

\subsubsection{Design Specifications}

Based on the design above, the multi-band ARC can hold for the following specifications shown in Table 1.

Table 1. Specifications of the multi-band ARC.

\begin{tabular}{|c|c|}
\hline Parameter & Value \\
\hline Frequency & $\mathrm{L} / \mathrm{C} / \mathrm{X} / \mathrm{Ka}$-bands \\
\hline Band & $200 \mathrm{MHz}$ (L-band), $240 \mathrm{MHz}$ (C-band), $1200 \mathrm{MHz}$ (X/Ka-bands) \\
\hline Radar cross section (RCS) & $35 \sim 55 \mathrm{dBsm}$ \\
\hline Range delay & $0.5 \mu \mathrm{s}, 1 \mu \mathrm{s}, 2 \mu \mathrm{s}$, \\
\hline RCS step & $5 \mathrm{~dB}$ \\
\hline Polarization Isolation & More than $40 \mathrm{~dB}$ \\
\hline RCS stability & $0.2 \mathrm{~dB}$ (L/C/X-bands), $0.3 \mathrm{~dB}$ (Ka-band) \\
\hline Gain flatness & 1 dBp_p (L/C/X-band), 3 dBp_p (Ka-band) \\
\hline Dynamic range & $\begin{array}{c}\text { L: approx. }-15.9 \mathrm{dBm} / \mathrm{m}^{2} \text { to }-69.9 \mathrm{dBm} / \mathrm{m}^{2} \\
\text { C: approx. }-3 \mathrm{dBm} / \mathrm{m}^{2} \text { to }-68 \mathrm{dBm} / \mathrm{m}^{2} \\
\text { X: approx. } 1.5 \mathrm{dBm} / \mathrm{m}^{2} \text { to }-58.5 \mathrm{dBm} / \mathrm{m}^{2} \\
\text { Ka: approx. }-9.25 \mathrm{dBm} / \mathrm{m}^{2} \text { to }-43.25 \mathrm{dBm} / \mathrm{m}^{2}\end{array}$ \\
\hline Polarization & H-pol (L-band), H and V-pol (C/X-bands), V-pol (Ka-band) \\
\hline Antenna pointing precision & Better than $0.2^{\circ}$ \\
\hline
\end{tabular}

Except for the fairly good specifications, the multi-band ARC is also designed for several operational modes such as the transponder mode, receiver mode, standby mode, self-calibration mode, and RCS calibration mode.

In the transponder mode, the active RCS setting is supported by a $5 \mathrm{~dB}$ step attenuator, and the accuracy of RCS is monitored and guaranteed by the gain calibration circuit. Moreover, tunable delay is possible in the range, which means that the point target response in the final image is not at the location of the ARC, which reduces the re-radiation effects of the structure of itself.

In receiver mode, the azimuth pattern of the SAR can be recorded by one ARC, and the elevation pattern of the SAR can also be acquired if some ARCs are placed in dedicated areas. The characteristics of the pulse transmitted by the SAR such as the amplitude flatness, the orthogonality, and the imbalance between the I and Q signals, the pulse width, and so on can also be measured and monitored in receiver mode. 
In standby mode, the control unit waits for the satellite to pass by. The control and data collection subsystem will be booted up at a pre-programmed wake-up time to power the whole ARC and set the proper operational mode.In self-calibration mode, the ARC will check the status itself and record the status information. Also, the gain will be calibrated to a standard to ensure the accuracy of RCS and the stability of the receiver.

In RCS calibration mode, an outside test range and a metal plate with a calculated radar cross section are used. A calibration pulse is transmitted from the ARC, reflected by the plate, received by the $\mathrm{ARC}$, and retransmitted after a $2 \mu$ s delay until the pulse is attenuated to a small enough size. A series of decaying pulses are monitored and recorded within the RF loop from which the absolute RCS of the ARC can be extracted. [22,23]

\subsection{Manufacture and Integration of the ARC}

Using the aforementioned design methods and specifications, we manufactured three multi-band ARCs to calibrate the Gapfen-3 SAR, Shenzhen-1 SAR, Luojia-2 SAR, and so on. Figure 6 is an outer view of the ARC. We can see from Figure 6 that the receiving and transmitting antennas, the radio frequency box (RF box), and the digital control box (DC box) were installed on the same flatform; therefore, there is no relative motion among the antenna, RF box, and DC box when the ARC works. So, the error due to cables connected to the antenna, RF box, and DC box is decreased a lot. Furthermore, $4 \mathrm{G}$ antennas for remote communication and a GPS/Beidou antenna for acquiring time were installed on the ARC.

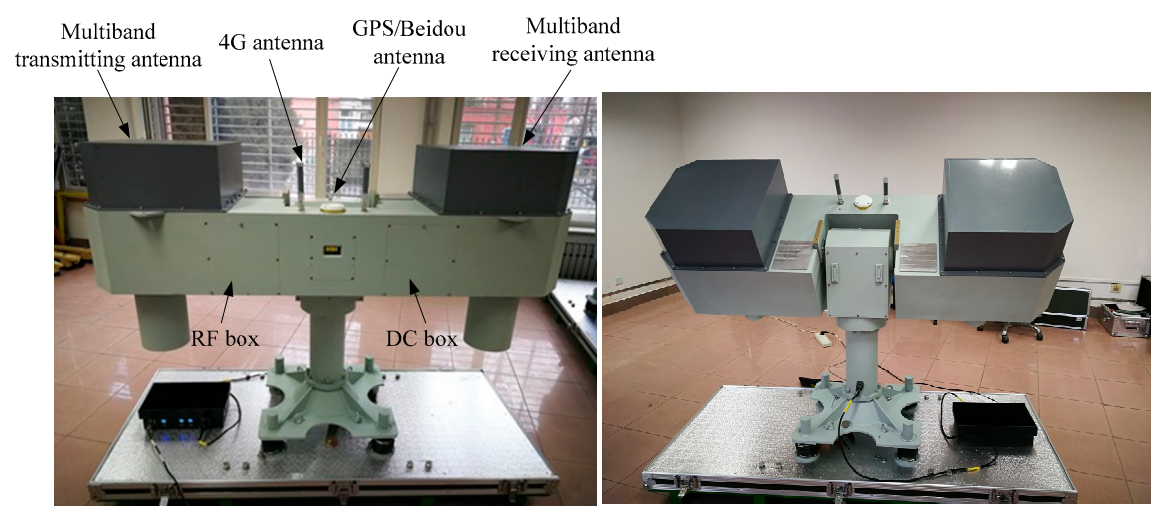

Figure 6. Outer view of the multi-band ARC.

Figure 7 shows an outer view of the manufactured multi-band antenna, and Figure 8 shows the patterns for the four bands measured in the anechoic chamber of Science and Technology on Antenna and Microwave Laboratory, Xidian University of China. We also show the cross-polarized pattern for comparison. We can see that the cross-polarization level is more than $30 \mathrm{~dB}$ lower than the co-polarization level for the L-band and Ka-band and $40 \mathrm{~dB}$ lower than the co-polarization level for the C-band and X-band.

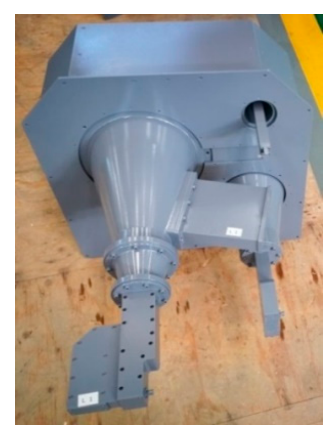

Figure 7. Outer view of the multi-band antenna. 


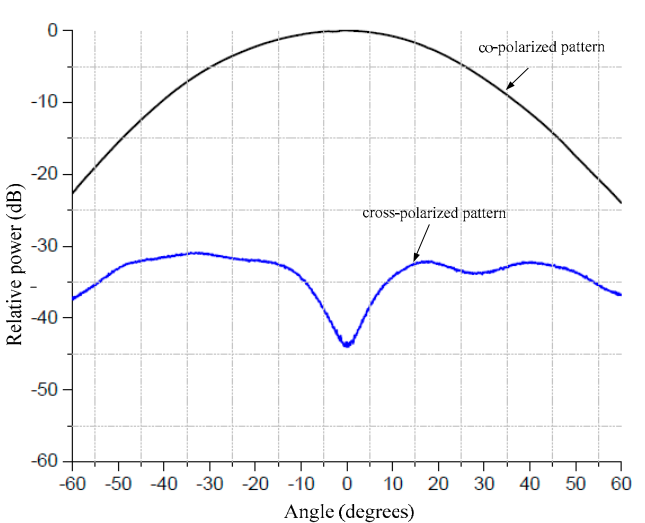

(a)

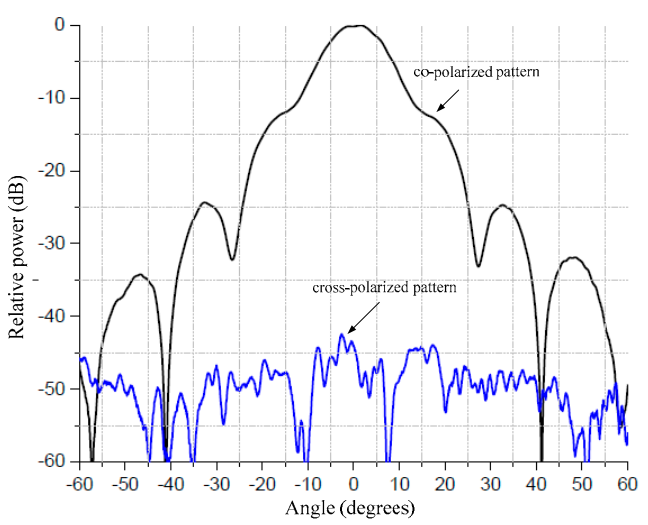

(c)

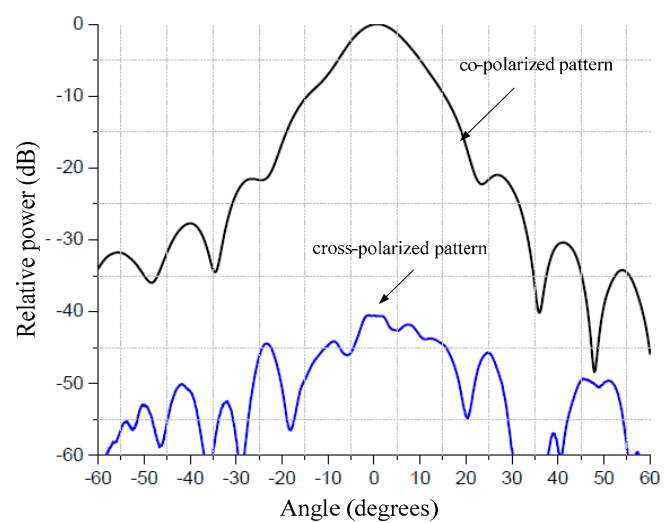

(b)

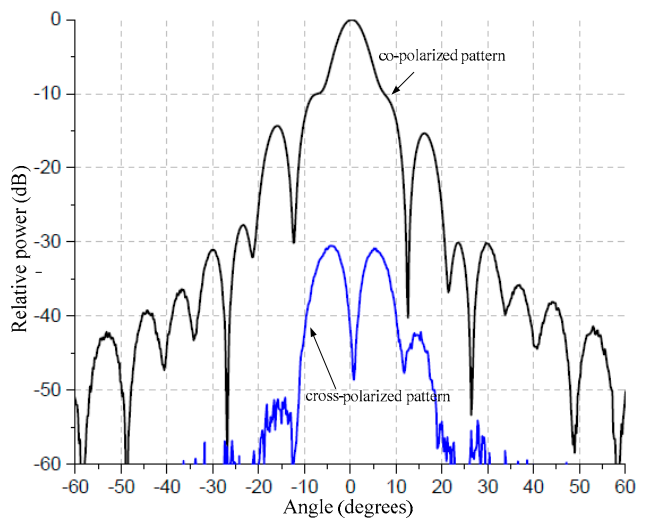

(d)

Figure 8. Examples of measured patterns: (a) L-band; (b) C-band; (c) X-band; (d) Ka-band.

The integration of ARC was performed after all the subsystems had been fulfilled. Actually, the main step in integration is the integration of the RF box and DC box. Figure 9 shows an outer view of the integration of the RF box and DC box on the table. During the integration of the RF box and DC box, the standard of the receiver gain and transponder gain were decided. After the integration of RF box and DC box, the RF box and DC box were installed on the ARC, and the RCS absolute calibration of ARC was done.

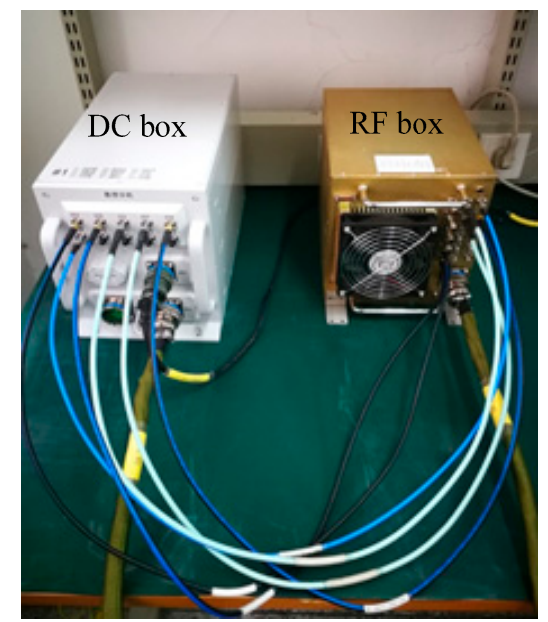

Figure 9. Outer view of the integration of the radio frequency (RF) box and digital control (DC) box on the table. 
Figure 10 is an outer view of the RF box (RF subsystem) for the multi-band ARC. We can see from Figure 10 that the RF subsystem is very complicated and that a modular design was used. The maximum gain is about $67 \mathrm{~dB}$ for the L-band, $55 \mathrm{~dB}$ for the C-band, $60 \mathrm{~dB}$ for the $\mathrm{X}$-band, and $64 \mathrm{~dB}$ for the Ka-band and these can be reduced by $15 \mathrm{~dB}$ in $5 \mathrm{~dB}$ steps. The gain can also be reduced by $20 \mathrm{~dB}$ using a voltage-tuned attenuator.

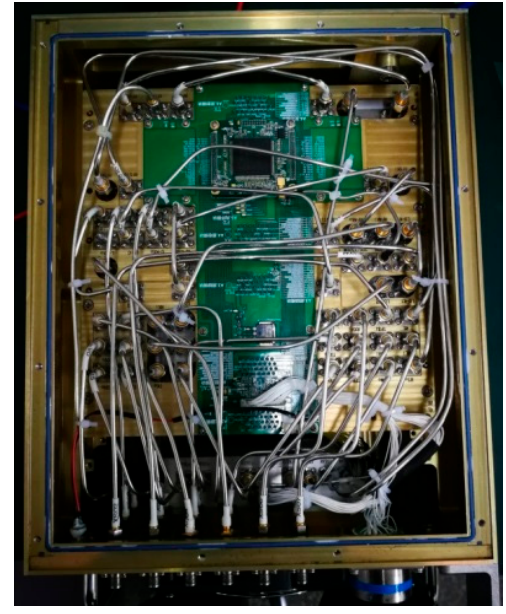

(a)

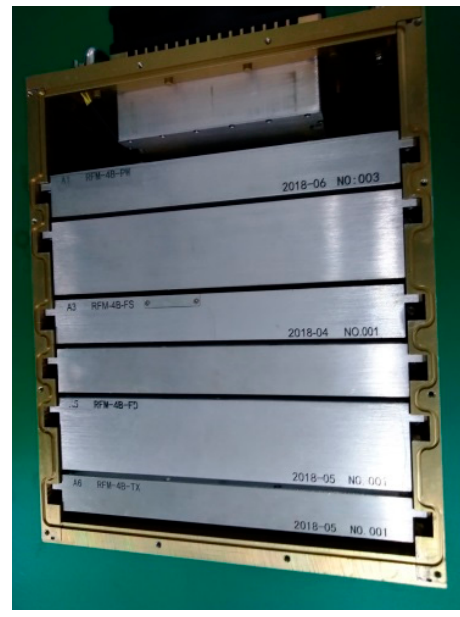

(b)

Figure 10. Outer view of the RF box: (a) one side; (b) the other side.

\subsection{Measurement of the ARC}

A set of lab measurements were performed in order to characterize the multi-band ARC at its operational frequencies. The following measurements were made.

\subsubsection{Gain Stability}

Based on the gain calibration method mentioned in the previous section, the gain stability is very good. We measured the gain of the multi-band ARC for about two weeks from 28 November to 14 December 2018. During these two weeks, we measured the gain several times every day using a vector network analyzer. Every measurement was made after the gain had been calibrated using our gain calibration circuit. Figure 11 shows the gain during the two weeks. It can be seen from Figure 11 that the gain stability is less than $\pm 0.2 \mathrm{~dB}$ for the L-band, $\pm 0.22 \mathrm{~dB}$ for the C-band, $\pm 0.21 \mathrm{~dB}$ for the X-band, and about $\pm 0.25 \mathrm{~dB}$ for the Ka-band. The gain is very stable over a long time, thus guaranteeing the RCS stability of the ARC.

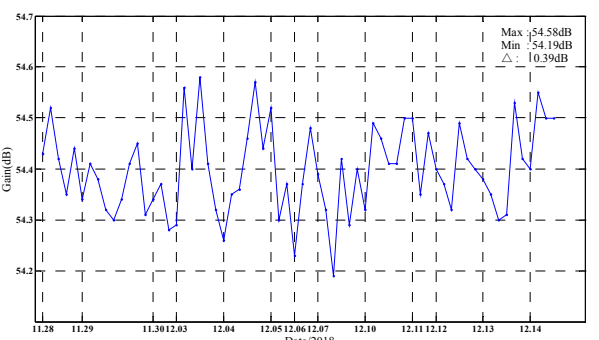

(a)

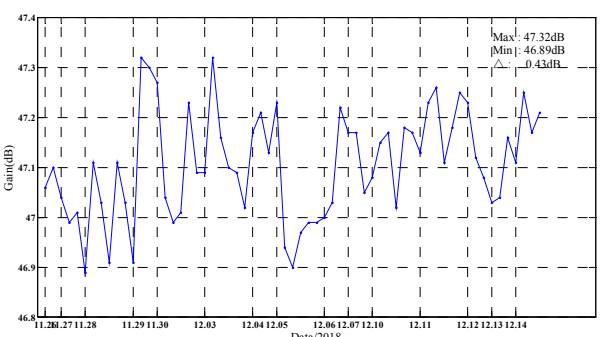

(b)

Figure 11. Cont. 


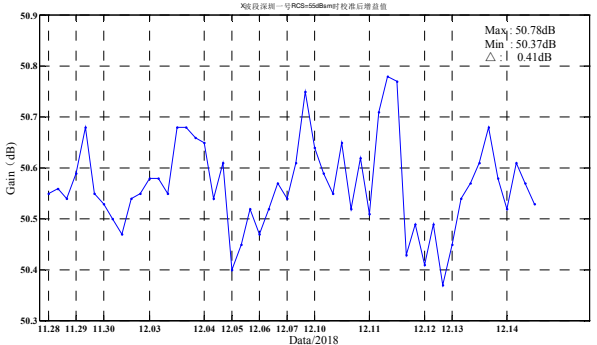

(c)

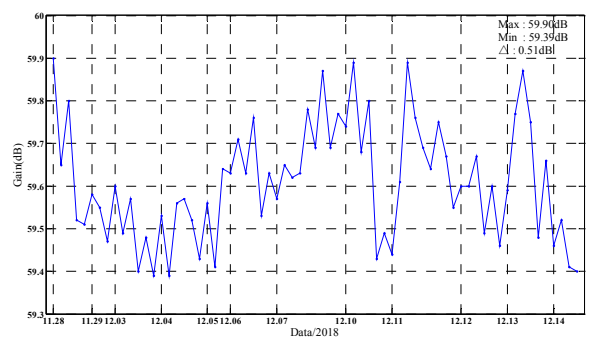

(d)

Figure 11. Gain during the two week period: (a) L-band; (b) C-band; (c) X-band; (d) Ka-band.

\subsubsection{System Transfer Function}

To evaluate the system transfer function, a measurement system consisting of a signal generator and a spectrum analyzer was set up [12]. The test signal was sent to the ARC by the signal generator and then to the spectrum analyzer. The amplitude of the signal spanned a range from $-60 \mathrm{dBm}$ to $-27 \mathrm{dBm}$ for the L-band, $-60 \mathrm{dBm}$ to $-17 \mathrm{dBm}$ for the C-band, $-60 \mathrm{dBm}$ to $-16 \mathrm{dBm}$ for the X-band, and $-70 \mathrm{dBm}$ to $-34 \mathrm{dBm}$ for the Ka-band, according to various parameters including the effective isotropic radiated power (EIRP), the incidence angle, and the orbit of the spaceborne SAR. Figure 12 gives the transfer functions for the four bands. From Figure 12, we calculated the linearity, which is better than $0.1 \mathrm{~dB}$ for the L-band, $0.15 \mathrm{~dB}$ for the C-band, $0.2 \mathrm{~dB}$ for the $\mathrm{X}$-band, and $0.5 \mathrm{~dB}$ for the Ka-band, and the linearity of the ARC is rather good.

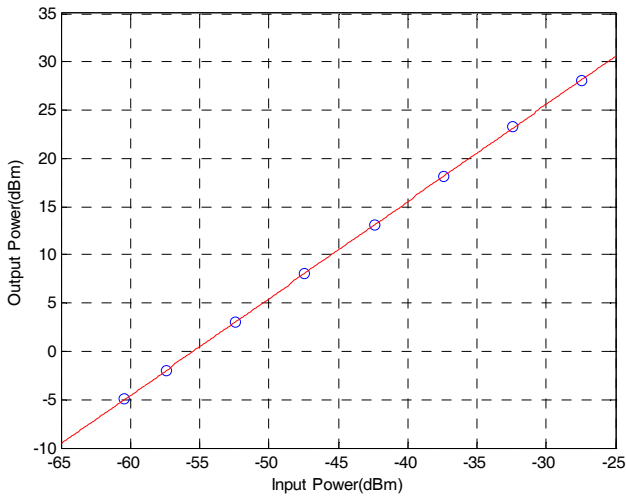

(a)

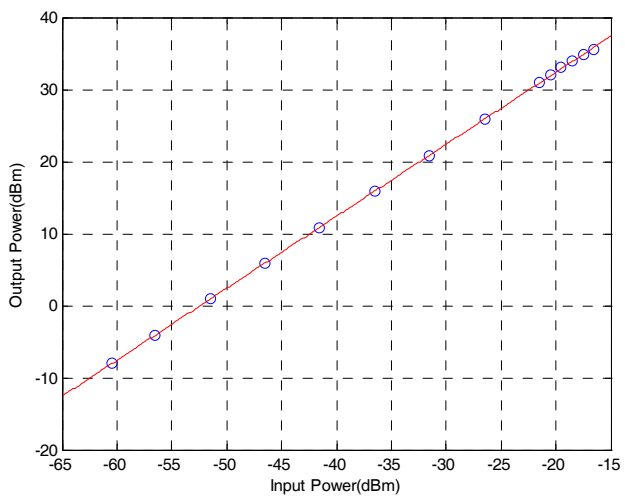

(c)

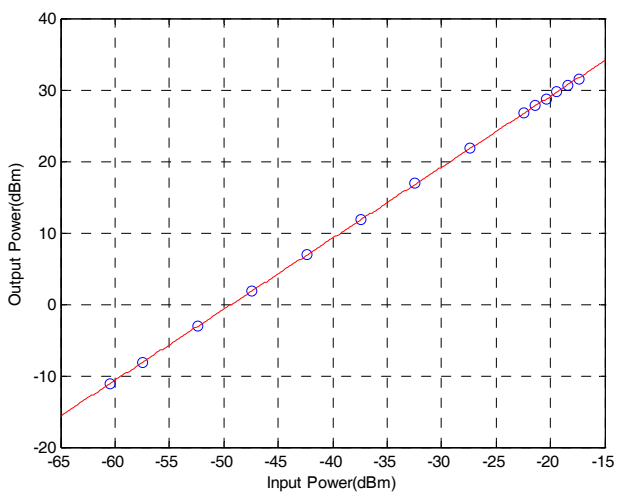

(b)

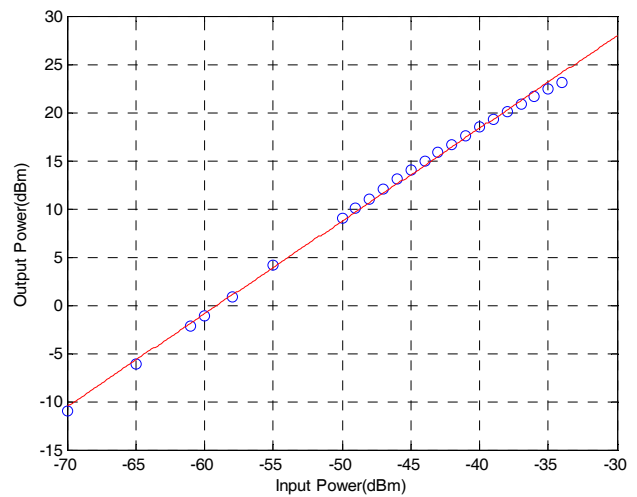

(d)

Figure 12. Transfer function: (a) L-band; (b) C-band; (c) X-band; (d) Ka-band. 


\subsubsection{Gain over Frequency Bandwidth}

This measurement allowed us to evaluate the working frequency bandwidth of ARC and the relative gain flatness. The inferior gain flatness influences the target image quality of the ARC. Many measures were taken to improve the specifications of the ARC. In Figure 13, the gain flatness for the four bands is shown. It can be seen from Figure 13 that the manufactured $A R C$ has gain flatness values of $\pm 0.5 \mathrm{~dB}$ (L-band), $\pm 0.2 \mathrm{~dB}$ (C-band), $\pm 0.5 \mathrm{~dB}$ (X-band), and $\pm 1.5 \mathrm{~dB}$ (Ka-band) over the available bandwidth of $200 \mathrm{MHz}$ for the L-band, $240 \mathrm{MHz}$ for the C-band, $1200 \mathrm{MHz}$ for the X-band, and 1200 $\mathrm{MHz}$ for the Ka-band, respectively.

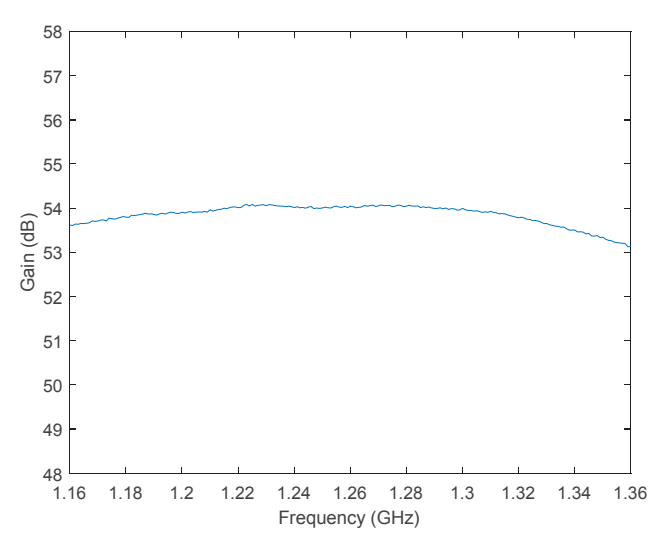

(a)

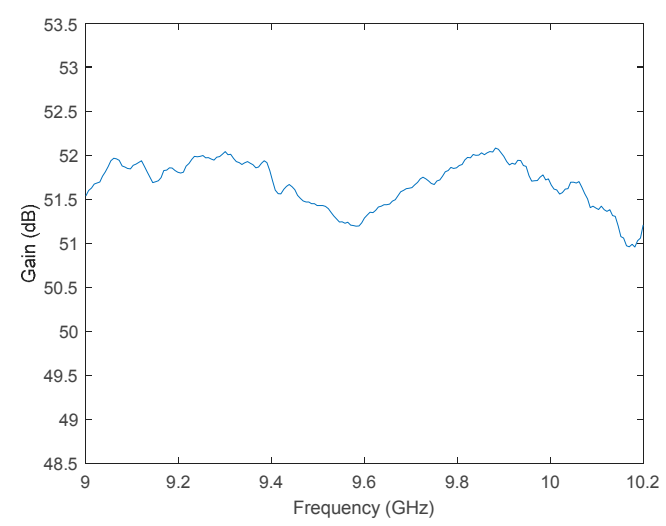

(c)

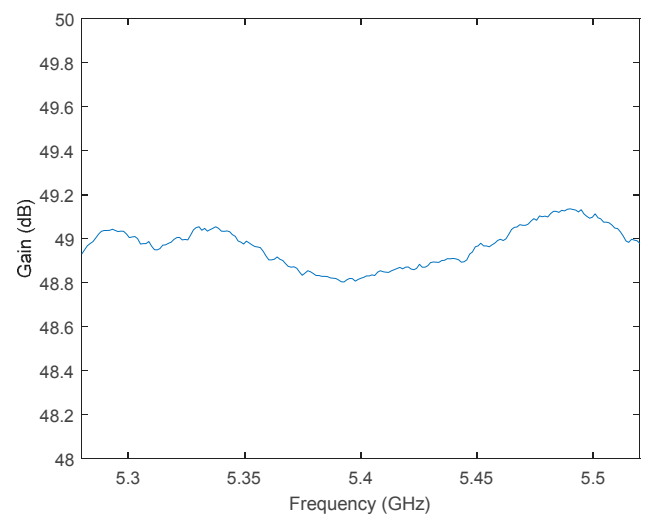

(b)

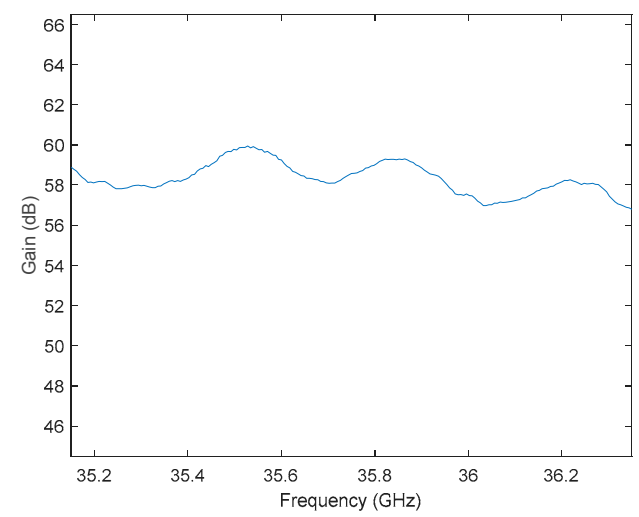

(d)

Figure 13. The gain flatness: (a) L-band; (b) C-band; (c) X-band; (d) Ka-band.

\subsubsection{Linearity of the ARC Receiver}

Linearity is one the key features of the ARC receiver [24]. After manufacturing the ARC, the linearity was measured using the signal generator. The signal was input into the ARC, and the ARC receiver collected the signal. The linearity errors for the full dynamic range were acquired from the collected data, and they are plotted in Figure 14. It can be seen from Figure 14 that the linearity errors are mostly below $0.2 \mathrm{~dB}$, which suggests very precise antenna pattern recognition, as the pattern is mathematically derived from the received power. 


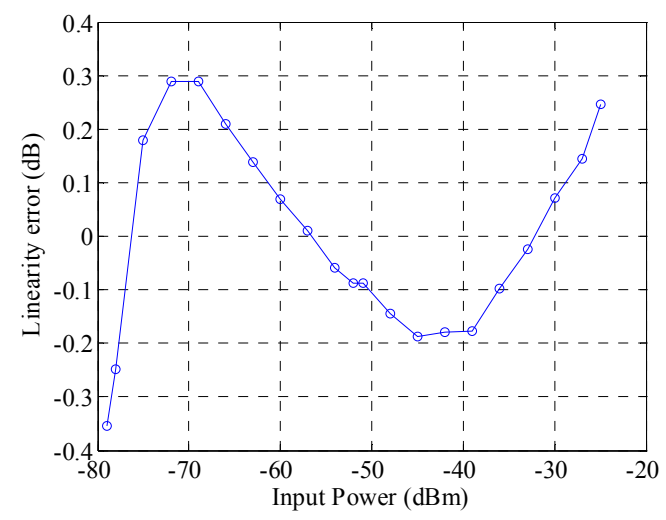

(a)

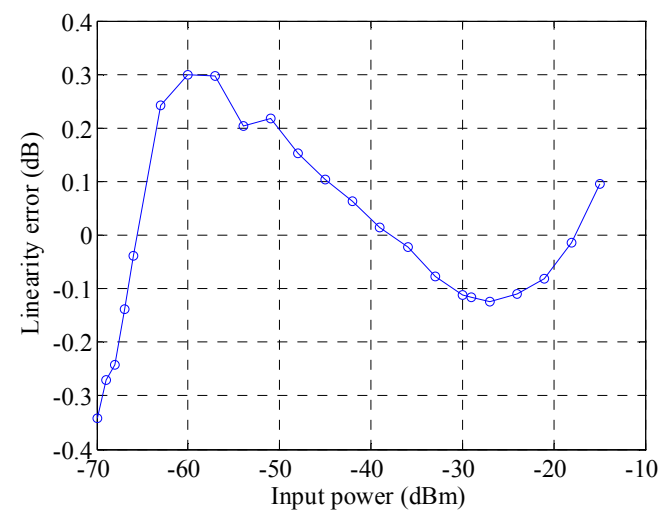

(c)

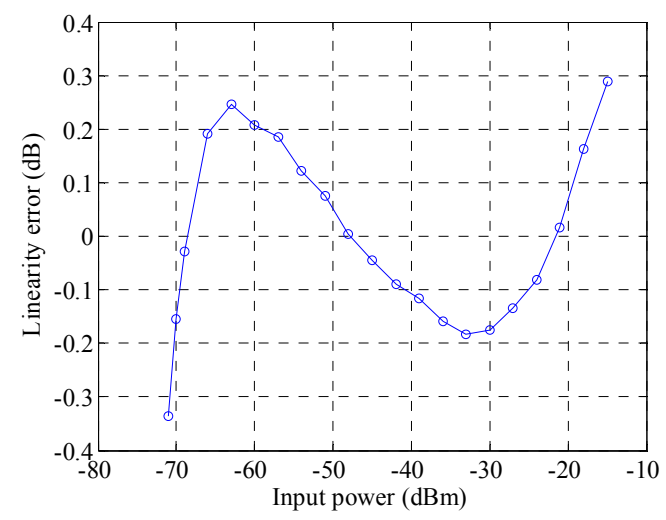

(b)

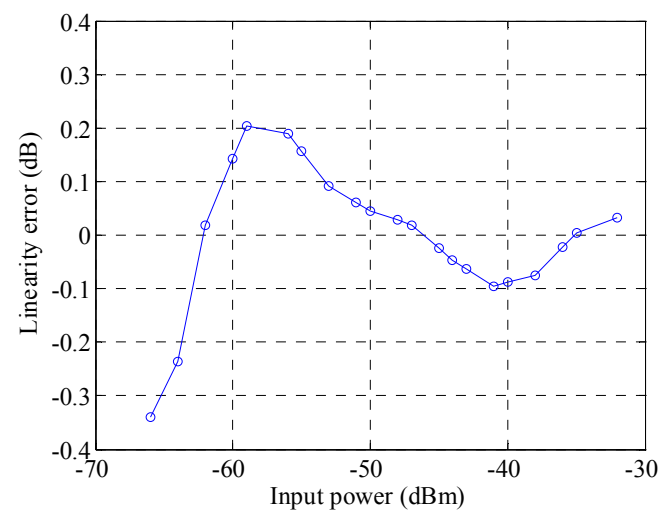

(d)

Figure 14. Linearity error for the full dynamic range: (a) L-band; (b) C-band; (c) X-band; (d) Ka-band.

\section{Discussion}

We developed a multi-band ARC that can calibrate L-, C-, X- and Ka-band spaceborne SARs, evaluate the image quality, and verify the calibration results. Although the multi-band ARC has many advantages, the system, especially the RF subsystem and antenna, is very complex, and it is rather expensive compared with a single-band ARC. Moreover, any piece of equipment has a given lifetime. Therefore, if the interval between the launch of various band spaceborne SARs is too long, we should consider using a single-band ARC for each SAR for the sake of cost and simplification. Of course, if there are already various band SARs in space, the use of the multi-band ARC will be very meaningful because on the one hand, the cost of a multi-band ARC will be much lower than the total cost of multiple single-band ARCs, and on the other hand, the number of ARC will be reduced a lot by using the multi-band ARC compared with the single-band ARC for each SAR, which will reduce the workload of the calibration campaign.

In this paper, we presented the results of the multi-band ARC. We found that the gain stability and the gain flatness of Ka-band were slightly worse than the other three bands, which is attributed to the very high frequency of the Ka-band, and the measurement error, which will be large. Furthermore, this paper only includes measurements conducted in the laboratory, and the experimental results for in-orbit calibration will be given after the satellite is launched in our future work.

\section{Conclusions}

This paper describes the principles, design, manufacture, and measurement results of a multi-band ARC working in the L-, C-, X- and Ka-bands. Multi-band and multi-functional antennas, for example, 
the L/C-band common aperture and the dual-polarized C-band, were used in the ARC for the first time. A multi-band gain calibration circuit with a thermal stability of better than $0.1 \mathrm{~dB}$ over the temperature range of -20 to $-50^{\circ} \mathrm{C}$ was designed to ensure a gain stability of about $0.2 \mathrm{~dB}$ for a significant period of time for all four operational frequency bands.

We manufactured three multi-band ARCs, and the gain stability, system transfer function, gain flatness, and linearity of ARC receiver were measured in the laboratory in order to characterize the multi-band ARC at its operational frequency bands. The results agreed well with the theoretical predictions and proved that the present multi-band $A R C$ has excellent performance. For example, the gain stability is better than $\pm 0.2 \mathrm{~dB}$ for the L-band, $\pm 0.22 \mathrm{~dB}$ for the C-band, $\pm 0.21 \mathrm{~dB}$ for the $\mathrm{X}$-band, and about $\pm 0.25 \mathrm{~dB}$ for the Ka-band for a significant period of time-about two weeks. These three multi-band ARCs will be used as the prime calibration standards during a validation and calibration campaign of the Shenzhen-1 SAR, Luojia-2 SAR, and other SARs after the launch of the satellites.

Author Contributions: L.L. and J.H. conceived and designed the experiments; L.L. and G.L. performed the experiments; L.L., F.M. and G.L. analyzed the data; L.L., F.M. and Y.W. contributed reagents/materials/analysis tools; L.L.wrote the paper.

Acknowledgments: This research was funded by the National Natural Science Foundation of China grant number 61571417; The authors thank the anonymous reviewers for their constructive comments and suggestions.

Conflicts of Interest: The authors declare no conflict of interest.

\section{References}

1. Touzi, R.; Hawkins, R.K.; Côté, S. High-precision assessment and calibration of polarimetric radarsat-2 SAR using transponder measurements. IEEE Trans. Geosci. Remote Sens. 2013, 51, 487-503. [CrossRef]

2. Lenz, R.; Pontes, J.; Wiesbeck, W. A high accuracy calibration and receive instrument for TerraSAR-X ground calibration. In Proceedings of the European Radar Conference, Paris, France, 3-4 October 2005; pp. 427-430.

3. Paul, S.; Evert, A.; Bjoern, R.; Nicolas, F.; Malcolm, D.; Berthyl, D. Transponder development for Sentinel-1. In Proceedings of the 8th European Conference on Synthetic Aperture Radar, Aachen, Germany, 7-10 June 2010; pp. 354-357.

4. Matthias, J.; Björn, D.; Daniel, R.; Sebastian, R.; Marco, S. Development of the highly accurate DLR Kalibri transponder. In Proceedings of the 10th European Conference on Synthetic Aperture Radar, Berlin, Germany, 3-5 June 2014; pp. 1176-1179.

5. Ming, F.; Hong, J.; Li, L. The external calibration system of GF-3 satellite. In Proceedings of the 12th European Conference on Synthetic Aperture Radar, Aachen, Germany, 4-6 June 2018; pp. 417-420.

6. Li, L.; Zhu, Y.T.; Hong, J.; Ming, F.; Wang, Y. Design and implementation of a novel polarimetric active radar calibrator for Gaofen-3 SAR. Sensors 2018, 18, 2620. [CrossRef] [PubMed]

7. Liang, W.B.; Jia, Z.Z.; Qiu, X.; Hong, J.; Zhang, Q.; Lei, B.; Zhang, F.; Deng, Z.; Wang, A. Polarimetric calibration of the GaoFen-3 mission using active radar calibrators and the applicable conditions of system model for radar polarimeters. Remote Sens. 2019, 11, 176. [CrossRef]

8. Woode, A.D.; Desnos, Y.L.; Jackson, H. The development and first results from the ESTEC ERS-1 active radar calibration unit. IEEE Trans. Geosci. Remote Sens. 1992, 30, 1122-1130. [CrossRef]

9. Fujita, M. Development of a retrodirective PARC for ALOS/PALSAR calibration. IEEE Trans. Geosci. Remote Sens. 2003, 4, 2177-2186. [CrossRef]

10. Li, L.; Hong, J.; Ming, F. Study on digital coded technology in active radar calibrator of SAR. In Proceedings of the International Geoscience and Remote Sensing Symposium, Munich, Germany, 22-27 July 2012; pp. 4509-4512.

11. Freeman, A.; Shen, Y.; Werner, C.L. Polarimetric SAR calibration experiment using active radar calibrators. IEEE Trans. Geosci. Remote Sens. 1990, 28, 224-240. [CrossRef]

12. Schena, V.D.; Posa, F.; Ponte, S.; De Carolis, G. Development and performance validation of L-, C- and X-band active radar calibrators (ARC) by means of laboratory tests and SIR-C/X-SAR experiments. In Proceedings of the International Geoscience and Remote Sensing Symposium, Firenze, Italy, 10-14 July 1995; pp. 80-82. 
13. Sarabandi, K.; Kashanianfard, M.; Nashashibi, A.Y.; Pierce, L.E.; Hampton, R. A polarimetric active transponder with extremely large RCS for absolute radiometric calibration of SMAP radar. IEEE Trans. Geosci. Remote Sens. 2018, 56, 1269-1277. [CrossRef]

14. Massimiliano, P.; Lapo, M. Cross-pol transponder with frequency shifter for bistatic ground-based synthetic aperture radar. Remote Sens. 2018, 10, 1364.

15. Zhang, X.X. Unified jamming equation of synthetic aperture radar. J. China Acad. Electron. Inf. Technol. 2006, 1, 107-113.

16. Skolnik, M. Introduction to Radar Systems; McGraw-Hill Education: New York, NY, USA, 2002.

17. Zhang, Q.; Yuan, C.W.; Liu, L. Radiation characteristics of L/C dual-band coaxial horn feed for high power microwaves. High Power Part. Beam. 2011, 1, 146-150. (In Chinese) [CrossRef]

18. Lin, W.Q.; Zhang, Z.Y.; Fu, G. Design of a high gain and low cross-polarization tri-band horn antenna. In Proceedings of the International Conference Microwave and Millimeter Wave Technology (ICMMT), Chengdu, China, 6-10 May 2018; pp. 1-3.

19. Li, L.; Hong, J.; Wang, Y.; Yi, M.; Zhu, Y.; Ming, F. In orbit calibration of GF-3 SAR using quad-polarized transponder. Acta Electron. Scin. 2017, 46, 2157-2164. (In Chinese)

20. Park, D.J.; Ahn, S.I.; Chun, Y.S.; Shin, J.M.; Yoon, J.C.; Kim, J.H. Development of active transponder for KOMPSAT-5 mission. In Proceedings of the 3rd International Asia-Pacific Conference on Synthetic Aperture Radar (APSAR), Seoul, Korea, 26-30 September 2011; pp. 794-797.

21. Jackson, H.D.; Alan, W. Development of the ERS-1 active radar calibration Unit. IEEE Trans. Microwave Theory Tech. 1992, 40, 1063-1069. [CrossRef]

22. Rudolf, D.; Raab, S.; Döring, B.J.; Jirousek, M.; Reimann, J.; Schwerdt, M. Absolute radiometric calibration of the novel DLR “Kalibri” transponder. In Proceedings of the Germany Microwave Conference, Nuremberg, Germany, 16-18 March 2015; pp. 323-326.

23. Rudolf, D.; Döring, B.J.; Matthias, J. Absolute radiometric calibration of C-Band transponders with proven plausibility. In Proceedings of the 10th European Conference on Synthetic Aperture Radar, Berlin, Germany, 3-5 June 2014; pp. 1180-1183.

24. Lenz, R.; Pontes, J.; Wiesbeck, W. The TerraSAR-X ground calibration system and pattern estimation software. In Proceedings of the International Geoscience and Remote Sensing Symposium, Seoul, Korea, 25-29 July 2005; pp. 4894-4897.

(C) 2019 by the authors. Licensee MDPI, Basel, Switzerland. This article is an open access article distributed under the terms and conditions of the Creative Commons Attribution (CC BY) license (http://creativecommons.org/licenses/by/4.0/). 